JOURNAL OF

FUNCTION SPACES AND APPLICATIONS

Volume 8, Number 1 (2008), 47-58
(C) 2008, Scientific Horizon http://www.jfsa.net

\title{
The Dirichlet Problem for elliptic equations in unbounded domains of the plane
}

\author{
Paola Cavaliere and Maria Transirico
}

(Communicated by Josip Pečarić)

2000 Mathematics Subject Classification. 35J25, 35R05.

Keywords and phrases. Elliptic equations, VMO-coefficients.

Abstract. In this paper we prove a uniqueness and existence theorem for the Dirichlet problem in $W^{2, p}$ for second order linear elliptic equations in unbounded domains of the plane. Here the leading coefficients are locally of class VMO and satisfy a suitable condition at infinity.

\section{Introduction}

Consider the Dirichlet problem

$$
\left\{\begin{array}{l}
u \in W^{2, p}(\Omega) \cap \stackrel{\circ}{W^{1, p}}(\Omega) \\
L u=f, \quad f \in L^{p}(\Omega)
\end{array}\right.
$$

where $\Omega$ is an open subset of $\mathbb{R}^{n}, n \geq 2$, with a suitable regularity property, $p \in] 1,+\infty[$, and $L$ is a second-order, linear, uniformly elliptic operator, i.e.

$$
L=-\sum_{i, j=1}^{n} a_{i j} \frac{\partial^{2}}{\partial x_{i} \partial x_{j}}+\sum_{i=1}^{n} a_{i} \frac{\partial}{\partial x_{i}}+a .
$$


If $n \geq 3, p<n$ and $\Omega$ is bounded, it is well known that the sole assumption

$$
a_{i j}=a_{j i} \in L^{\infty}(\Omega)
$$

(together with suitable summability conditions for the coefficients $a_{i}$ and $a$ ) is not enough to ensure the uniqueness for the problem (1.1). It has been a challenging problem to determine suitable additional hypotheses, in terms of discontinuity, on the leading coefficients $a_{i j}$ in order to get the well-posedness of (1.1). In particular, Miranda [13] proved that it holds whenever $p=2$ and the coefficients $a_{i j}$ belong to $W^{1, n}(\Omega)$; later generalizations required the derivatives of the leading coefficients to belong to certain wider spaces. Recently, in two profound papers [9] and [10], it has been pointed out that the solvability of (1.1) holds, for every $p \in] 1,+\infty[$, under the assumption that the $a_{i j}$ 's are of class $V M O, a_{i}=a=0$ (note that $V M O$ contains $\left.W^{1, n}(\Omega)\right)$ and, later, the condition $a_{i}=a=0$ has been removed in [19] and [20].

If the set $\Omega$ is unbounded, problem (1.1) has for instance been studied in [16], where the leading coefficients satisfy restrictions similar to those of [13] and $p=2$, in [3], where the investigation has been extended to the case $p \in] 1,+\infty$ [, and in [4] and [5], where the $a_{i j}$ 's verify assumptions similar to those of [9] and [10].

In the planar case $(n=2)$ and whenever $\Omega$ is bounded, Talenti [15] proved that, if $p=2$, condition (1.3) is enough to establish certain estimates for the solutions of (1.1) which lead to an existence and uniqueness result. Further studies have shown that this result still holds even if $p$ lies in a certain neighborhood of 2 (see for instance [12]), and this interval has recently been determined in [2]. Observe that the lower critical exponent here is precisely the one conjectured by C. Pucci [14], who also proved that if $p$ is smaller than this exponent, then the uniqueness of the solution of (1.1) cannot be proved. In our previous paper [7] we have studied problem (1.1) when $p$ is an arbitrary real number $>1$ and the $a_{i j}$ 's belong to $V M O(\Omega)$.

The aim of the present one is to extend the investigation of [7] to the case of unbounded open sets. In fact, under the hypotheses that the coefficients $a_{i j}$ are in $V M O_{\text {loc }}(\bar{\Omega})$ and satisfy a suitable condition at infinity, some $W^{2, p}$-estimates for the solutions of (1.1) and some regularity results will be obtained, and an existence and uniqueness theorem will be deduced.

\section{Preliminaries}

In this section we collect the notation as well as the definitions of function spaces being used throughout the paper. 
Let $\Omega$ be an open subset of $\mathbb{R}^{n}$, and let $\Sigma(\Omega)$ be the collection of all Lebesgue measurable subsets of $\Omega$. For each $E \in \Sigma(\Omega),|E|$ and $\chi_{E}$ denote the Lebesgue measure and the characteristic function of $E$, respectively. Moreover,

$$
E(x, \rho)=E \cap B(x, \rho), \quad x \in \mathbb{R}^{n}, \rho \in \mathbb{R}_{+},
$$

for any open ball $B(x, \rho)$ in $\mathbb{R}^{n}$ of center $x$ and radius $\rho$. For convenience, we write

$$
B_{\rho}=B(0, \rho), \quad \rho \in \mathbb{R}_{+} .
$$

If $X(\Omega)$ is a space of functions defined on $\Omega$, we denote as $X_{\text {loc }}(\bar{\Omega})$ the space of all functions $g: \Omega \rightarrow \mathbb{R}$ such that $\zeta g \in X(\Omega)$ for every $\zeta \in \mathfrak{D}(\bar{\Omega})$, where $\mathfrak{D}(\bar{\Omega})=\left\{\xi_{\mid \Omega} \mid \xi \in C_{\circ}^{\infty}\left(\mathbb{R}^{n}\right)\right\}$.

For $p \in\left[1,+\infty\left[, M^{p}(\Omega)\right.\right.$ is the set of all functions $g \in L_{\mathrm{loc}}^{p}(\bar{\Omega})$ such that

$$
\|g\|_{M^{p}(\Omega)}=\sup _{x \in \Omega}\|g\|_{L^{p}(\Omega(x, 1))}<+\infty
$$

endowed with the norm defined by $(2.1)$ and $\tilde{M}^{p}(\Omega)$ (resp. $\left.M_{0}^{p}(\Omega)\right)$ is the closure of $L^{\infty}(\Omega)$ (resp. $C_{\circ}^{\infty}(\Omega)$ ) in $M^{p}(\Omega)$. It is known that a function $g$ (in $M^{p}(\Omega)$ ) belongs to $\tilde{M}^{p}(\Omega)$ if and only if the function

$$
\tau_{g}(t)=\sup _{\substack{E \in \Sigma(\Omega) \\ \sup _{x \in \Omega}|E(x, 1)| \leq t}}\left\|\chi_{E} g\right\|_{M^{p}(\Omega)}, \quad t \in \mathbb{R}_{+},
$$

vanishes when $t$ goes to zero. Then a modulus of continuity of $g$ in $\tilde{M}^{p}(\Omega)$ is a map $\tilde{\sigma}_{p}[g]: \mathbb{R}_{+} \rightarrow \mathbb{R}_{+}$such that

$$
\tilde{\sigma}_{p}[g](t) \geq \tau_{g}(t), \quad t \in \mathbb{R}_{+}, \quad \lim _{t \rightarrow 0^{+}} \tilde{\sigma}_{p}[g](t)=0 .
$$

If $\Omega$ has the property

$$
\left.\left.|\Omega(x, \rho)| \geq A \rho^{n}, \quad x \in \Omega, \rho \in\right] 0,1\right],
$$

where $A$ is some positive constant independent of $x$ and $\rho$, one can consider the space $B M O(\Omega, t), t \in \mathbb{R}_{+}$, consisting of all functions $g$ in $L_{\text {loc }}^{1}(\bar{\Omega})$ such that

$$
[g]_{B M O(\Omega, t)}=\sup _{\substack{x \in \Omega \\ \rho \in j 0, t]}} f_{\Omega(x, \rho)}\left|g-f_{\Omega(x, \rho)} g\right|<+\infty
$$

where

$$
f_{\Omega(x, \rho)} g=|\Omega(x, \rho)|^{-1} \int_{\Omega(x, \rho)} g
$$


A function $g \in B M O(\Omega)=B M O\left(\Omega, t_{A}\right)$, where

$$
t_{A}=\sup \left\{t \in \mathbb{R}_{+}: \sup _{\substack{x \in \Omega \\ \rho \in] 0, t]}} \frac{\rho^{n}}{|\Omega(x, \rho)|} \leq \frac{1}{A}\right\},
$$

is said to belong to the class $V M O(\Omega)$ if

$$
\lim _{t \rightarrow 0^{+}}[g]_{B M O(\Omega, t)}=0 .
$$

Then, a function $\eta[g]: \mathbb{R}_{+} \rightarrow \mathbb{R}_{+}$is called a modulus of continuity of $g$ in $V M O(\Omega)$ if

$$
\eta[g](t) \geq[g]_{B M O(\Omega, t)}, t \in \mathbb{R}_{+}, \quad \lim _{t \rightarrow 0^{+}} \eta[g](t)=0 .
$$

For a more detailed account of properties of the above function spaces we refer to [16], [17] and [18].

\section{Some a priori estimates}

Let $\Omega$ be an unbounded open set in $\mathbb{R}^{2}$ satisfying the $C^{1,1}$-regularity property, namely

$\left(\mathcal{P}_{\Omega}\right)$ there exist a locally finite open covering $\left(U_{i}\right)_{i \in \mathbb{N}}$ of $\partial \Omega$ and $C^{1,1}$ diffeomorphisms $\Phi_{i}: U_{i} \rightarrow B_{1}, i \in \mathbb{N}$, such that

- $\{x \in \Omega$ : $\operatorname{dist}(x, \partial \Omega)<\delta\} \subset \bigcup_{i \in \mathbb{N}} \Phi_{i}^{-1}\left(B_{1 / 2}\right)$ for some $\delta>0$;

- $\Phi_{i}\left(U_{i} \cap \Omega\right)=\left\{x \in B_{1}: x_{2}>0\right\}$ for each $i \in \mathbb{N}$;

- there is $m_{0} \in \mathbb{N}$ such that the intersection of any $m_{0}+1$ distinct $U_{i}$ 's is empty;

- the components of $\Phi_{i}$ and $\Phi_{i}^{-1}$ have bounded $C^{1,1}$-norms independently of $i$.

Notice that any open set $\Omega$ with the property $\left(\mathcal{P}_{\Omega}\right)$ also satisfies the condition (2.3) above.

From this point on, we assume that $p \in] 1,+\infty[, L$ is an elliptic operator in $\Omega$ of the form

$$
L=-\sum_{i, j=1}^{2} a_{i j} \frac{\partial^{2}}{\partial x_{i} \partial x_{j}}+\sum_{i=1}^{2} a_{i} \frac{\partial}{\partial x_{i}}+a
$$


and

$$
L_{0}=-\sum_{i, j=1}^{2} a_{i j} \frac{\partial^{2}}{\partial x_{i} \partial x_{j}}
$$

such that

$\left(h_{1}\right) \quad\left\{\begin{array}{l}a_{i j}=a_{j i} \in L^{\infty}(\Omega), \quad i, j=1,2, \\ \exists \nu \in \mathbb{R}_{+}: \sum_{i, j=1}^{2} a_{i j} \xi_{i} \xi_{j} \geq \nu|\xi|^{2} \text { a.e. in } \Omega, \forall \xi \in \mathbb{R}^{2},\end{array}\right.$

$\left(h_{2}\right) \quad\left\{\begin{array}{l}a_{i} \in \tilde{M}^{r}(\Omega), i=1,2, \text { where } \\ r=2 \text { if } p<2, r>2 \text { if } p=2, r=p \text { if } p>2, \\ a \in \tilde{M}^{p}(\Omega),\end{array}\right.$

$$
a_{i j} \in \operatorname{VMO}(\Omega), \quad i, j=1,2 .
$$

We stress that, under the assumptions $\left(h_{1}\right)$ and $\left(h_{2}\right)$, the operator

$$
L: W^{2, p}(\Omega) \rightarrow L^{p}(\Omega)
$$

is bounded (see e.g. Theorem 3.1 in [11]).

The next lemma provides an a priori bound.

Lemma 3.1. Assume $\left(h_{1}\right),\left(h_{2}\right)$ and $\left(h_{3}\right)$. If $u$ is a solution of the Dirichlet problem

$$
u \in W_{\mathrm{loc}}^{2, p}(\bar{\Omega}) \cap \stackrel{\circ}{W^{1, p}}(\Omega), \quad L u \in L^{p}(\Omega),
$$

then $u \in W^{2, p}(\Omega)$. Moreover

$$
\|u\|_{W^{2, p}(\Omega)} \leq c\left(\|L u\|_{L^{p}(\Omega)}+\|u\|_{L^{p}(\Omega)}\right),
$$

where the constant $c$ depends on $\Omega, p, r, \nu,\left\|a_{i j}\right\|_{L^{\infty}(\Omega)}, \eta\left[p\left(a_{i j}\right)\right],\left\|a_{i}\right\|_{M^{r}(\Omega)}$, $\|a\|_{M^{p}(\Omega)}, \tilde{\sigma}_{r}\left[a_{i}\right], \tilde{\sigma}_{p}[a]$, and $p\left(a_{i j}\right)$ is an extension of $a_{i j}$ to $\mathbb{R}^{2}$ of class $L^{\infty}\left(\mathbb{R}^{2}\right) \cap \operatorname{VMO}\left(\mathbb{R}^{2}\right)$.

Proof. Let us fix $u \in W_{\text {loc }}^{2, p}(\bar{\Omega}) \cap \stackrel{\circ}{W^{1, p}}(\Omega)$ such that $L u \in L^{p}(\Omega)$. The regularity hypothesis on $\Omega$ implies that there exists $d \in] 0,1[$ such that, for any $x \in \Omega, B(x, d) \subset \Omega$ or $B(x, d) \subset U_{i}$ for some $i \in I$. So, if $\zeta$ is a function of class $C_{\circ}^{\infty}\left(\mathbb{R}^{2}\right)$ such that 


$$
0 \leq \zeta \leq 1, \quad \zeta=1 \text { in } B\left(0, \frac{d}{2}\right), \quad \operatorname{supp} \zeta \subset B(0, d),
$$

for any fixed $x \in \Omega$, the function

$$
\psi=\psi_{x}: y \in \Omega \rightarrow \zeta(y-x)
$$

is obviously in $C_{\circ}^{\infty}\left(\mathbb{R}^{2}\right)$,

$$
0 \leq \psi \leq 1, \quad \psi=1 \text { in } B\left(x, \frac{d}{2}\right), \quad \operatorname{supp} \psi \subset B(x, d),
$$

and

$$
\psi u \in W^{2, p}(\Omega) \cap \stackrel{\circ}{W}^{1, p}(\Omega), \quad \operatorname{supp}(\psi u) \subset B(x, d) \cap \bar{\Omega} .
$$

Then from Lemma 3.1 in [7] it follows that

$$
\|\psi u\|_{W^{2, p}(\Omega(x, d))} \leq c_{1}\left(\|L(\psi u)\|_{L^{p}(\Omega(x, d))}+\|\psi u\|_{L^{p}(\Omega(x, d))}\right),
$$

where the constant $c_{1}$ depends on $\Omega, p, \nu,\left\|a_{i j}\right\|_{L^{\infty}(\Omega)}, \eta\left[p\left(a_{i j}\right)\right],\left\|a_{i}\right\|_{M^{r}(\Omega)}$, $\|a\|_{M^{p}(\Omega)}, \tilde{\sigma}_{r}\left[a_{i}\right]$ and $\tilde{\sigma}_{p}[a]\left(p\left(a_{i j}\right)\right.$ denotes an extension of $a_{i j}$ to $\mathbb{R}^{2}$ of class $L^{\infty}\left(\mathbb{R}^{2}\right) \cap V M O\left(\mathbb{R}^{2}\right)$; see Theorem 5.1 in [18] for the existence of such extension).

Since

$$
L(\psi u)=\psi L u-2 \sum_{i, j=1}^{2} a_{i j} u_{x_{i}} \psi_{x_{j}}+u\left(-\sum_{i, j=1}^{2} a_{i j} \psi_{x_{i} x_{j}}+\sum_{i=1}^{2} a_{i} \psi_{x_{i}}\right)
$$

from (3.5) and (3.6) we deduce the bound

$$
\begin{aligned}
\|u\|_{W^{2, p}\left(\Omega\left(x, \frac{d}{2}\right)\right)} \leq c_{2}( & \|L u\|_{L^{p}(\Omega(x, d))}+\left\|u_{x}\right\|_{L^{p}(\Omega(x, d))} \\
& \left.+\|u\|_{L^{p}(\Omega(x, d))}+\sum_{i=1}^{2}\left\|a_{i} \psi_{x_{i}} u\right\|_{L^{p}(\Omega(x, d))}\right),
\end{aligned}
$$

with $c_{2}$ depending on the same parameters as $c_{1}$. On the other hand, an application of Theorem 3.1 in [11] yields

$$
\left\|a_{i} \psi_{x_{i}} u\right\|_{L^{p}(\Omega(x, d))}=\left\|a_{i} \psi_{x_{i}} u\right\|_{L^{p}(\Omega)} \leq c_{3}\left\|a_{i}\right\|_{M^{r}(\Omega)}\left\|\psi_{x_{i}} u\right\|_{W^{1, p}(\Omega)},
$$

where $c_{3}$ depends only on $p$ and $r$. Therefore, from (3.7) and (3.8), one has that 


$$
\|u\|_{W^{2, p}\left(\Omega\left(x, \frac{d}{2}\right)\right)} \leq c_{4}\left(\|L u\|_{L^{p}(\Omega(x, d))}+\left\|u_{x}\right\|_{L^{p}(\Omega(x, d))}+\|u\|_{L^{p}(\Omega(x, d))}\right),
$$

with $c_{4}$ depending on the same parameters as $c_{2}$ and on $r$. By (3.9) and by Lemma 4.1 in [16] we obtain that $u \in W^{2, p}(\Omega)$ and

$$
\|u\|_{W^{2, p}(\Omega)} \leq c_{5}\left(\|L u\|_{L^{p}(\Omega)}+\left\|u_{x}\right\|_{L^{p}(\Omega)}+\|u\|_{L^{p}(\Omega)}\right),
$$

where $c_{5}$ depends on the same parameters on which $c_{4}$ does. Finally, there exists $K=K(p, \Omega) \in \mathbb{R}_{+}$such that

$$
\left\|u_{x}\right\|_{L^{p}(\Omega)} \leq K \varepsilon\|u\|_{W^{2, p}(\Omega)}+\frac{K}{\varepsilon}\|u\|_{L^{p}(\Omega)},
$$

for any $\varepsilon \in] 0,1]$ (see e.g. [1], Theorem 4.14). This combined with (3.10) gives (3.4).

Consider now the following hypotheses on the coefficients of $L$ :

$$
a_{i j} \in V M O_{\mathrm{loc}}(\bar{\Omega}), \quad i, j=1,2,
$$

there exist functions $\alpha_{i j}, i, j=1,2, g$ and $\mu \in \mathbb{R}_{+}$such that

$\left(h_{4}\right)$

$$
\left\{\begin{array}{l}
\alpha_{i j}=\alpha_{j i} \in L^{\infty}(\Omega) \cap V M O(\Omega), \quad i, j=1,2, \\
\sum_{i, j=1}^{2} \alpha_{i j} \xi_{i} \xi_{j} \geq \mu|\xi|^{2} \text { a.e. in } \Omega, \quad \forall \xi \in \mathbb{R}^{2}, \\
g \in L^{\infty}(\Omega), \\
\lim _{\rho \rightarrow+\infty} \sum_{i, j=1}^{2}\left\|\alpha_{i j}-g a_{i j}\right\|_{L^{\infty}\left(\Omega \backslash B_{\rho}\right)}=0 .
\end{array}\right.
$$

We point out that $\left(h_{3}^{\prime}\right)-\left(h_{4}\right)$ are weaker than $\left(h_{3}\right)$, as an example in [4] shows. The next result provides another a priori estimate, but under these weaker assumptions. In the claim, for any $\rho \in \mathbb{R}_{+}, \zeta_{\rho}$ stands for a function of class $C_{\circ}^{\infty}\left(\mathbb{R}^{2}\right)$ such that

$$
0 \leq \zeta_{\rho} \leq 1, \quad \zeta_{\rho}=1 \text { in } B_{\rho}, \quad \operatorname{supp} \zeta_{\rho} \subset B_{2 \rho} .
$$

Theorem 3.2. Assume $\left(h_{1}\right),\left(h_{2}\right),\left(h_{3}^{\prime}\right)$ and $\left(h_{4}\right)$. Then there exist positive real numbers $\rho_{0}, c$ such that

$$
\|u\|_{W^{2, p}(\Omega)} \leq c\left(\|L u\|_{L^{p}(\Omega)}+\|u\|_{L^{p}(\Omega)}\right), \quad \forall u \in W^{2, p}(\Omega) \cap \stackrel{\circ}{W^{1, p}}(\Omega),
$$


where $c$ depends only on $\Omega, p, r, \nu, \mu,\left\|a_{i j}\right\|_{L^{\infty}(\Omega)},\left\|\alpha_{i j}\right\|_{L^{\infty}(\Omega)},\|g\|_{L^{\infty}(\Omega)}$, $\eta\left[p\left(\zeta_{2 \rho_{0}} a_{i j}\right)\right], \eta\left[p\left(\alpha_{i j}\right)\right], \tilde{\sigma}_{r}\left[a_{i}\right], \tilde{\sigma}_{p}[a]$.

Proof. The proof is similar to the one given in [4] (Theorem 3.1), taking into account to apply our Lemma 3.1 in place of Theorem 5.1 in [6].

\section{Some regularity results}

The aim of this section is to prove two regularity results, the first one of local type and the other of global type. Note that for the local regularity case, we assume only the conditions $\left(h_{1}\right)$ and $\left(h_{3}^{\prime}\right)$ on the leading coefficients, while for the coefficients of lower order the condition $\left(h_{2}\right)$ is replaced by the weaker assumption

$\left(h_{2}^{\prime}\right) \quad\left\{\begin{array}{l}a_{i} \in L_{\mathrm{loc}}^{r}(\bar{\Omega}), i=1,2, \\ \text { where } r>2 \text { if } p \leq 2, r=p \text { if } p>2, \\ a \in L_{\mathrm{loc}}^{p}(\bar{\Omega}) .\end{array}\right.$

The following result is proved.

Lemma 4.1. Assume $\left(h_{1}\right),\left(h_{2}^{\prime}\right)$ and $\left(h_{3}^{\prime}\right)$. If $u$ is a solution of the problem

$$
\left\{\begin{array}{l}
u \in W_{\mathrm{loc}}^{2, q}(\bar{\Omega}) \cap \stackrel{\circ}{W_{\mathrm{loc}}^{1, q}(\bar{\Omega}),} \\
L u \in L_{\mathrm{loc}}^{p}(\bar{\Omega}),
\end{array}\right.
$$

where $q \in] 1, p]$, then $u$ belongs to $W_{\mathrm{loc}}^{2, p}(\bar{\Omega})$.

Proof. We may assume that $q<p$. Let $k$ be the positive integer such that

$$
k-1<2\left(\frac{1}{q}-\frac{1}{p}\right) \leq k,
$$

so that

$$
\frac{1}{q}-\frac{h}{2}>\frac{1}{p}, \quad h=0, \ldots, k-1, \quad \frac{1}{q}-\frac{k}{2} \leq \frac{1}{p} .
$$

Put

$$
\frac{1}{q_{h}}=\frac{1}{q}-\frac{h}{2}, \quad h=0, \ldots, k-1, \quad \frac{1}{q_{k}}=\frac{1}{p} .
$$

In order to prove the claim it is enough to show that

$$
L(\zeta u) \in L^{q_{1}}(\Omega), \quad \zeta \in \mathcal{D}(\bar{\Omega}),
$$


since by (4.4) and by Lemma 3.3 in [7], it will follow that $u \in W_{\text {loc }}^{2, q_{1}}(\bar{\Omega})$, and so the statement can be deduced iterating the above argument $k$ times.

Fixed $\zeta \in \mathcal{D}(\bar{\Omega})$, since

$$
\begin{aligned}
& L(\zeta u)=L_{0}(\zeta u)+\sum_{i=1}^{2} a_{i}(\zeta u)_{x_{i}}+a \zeta u \\
& =\zeta L u+u L_{0} \zeta-2 \sum_{i, j=1}^{2} a_{i j} u_{x_{i}} \zeta_{x_{j}}+u \sum_{i=1}^{2} a_{i} \zeta_{x_{i}},
\end{aligned}
$$

it is enough to check that

$$
u \zeta_{x_{i} x_{j}}, \quad u_{x_{i}} \zeta_{x_{j}}, \quad a_{i} u \zeta_{x_{i}} \in L^{q_{1}}(\Omega), \quad i, j=1,2 .
$$

By Sobolev embedding, one obtains that $u \zeta_{x_{i} x_{j}}, u_{x_{i}} \zeta_{x_{j}}$ belong to $L^{q_{1}}$; on the other hand, from Theorem 3.1 in [11] we can conclude that $a_{i} u \zeta_{x_{i}}$ also lies in $L^{q_{1}}$, and so (4.6) holds.

We prove now the following regularity result of global type.

Lemma 4.2. Assume $\left(h_{1}\right),\left(h_{2}\right)$ (with $\left.r>2\right),\left(h_{3}^{\prime}\right)$ and $\left(h_{4}\right)$. If $u$ is a solution of the problem

$$
\left\{\begin{array}{l}
u \in W_{\mathrm{loc}}^{2, q}(\bar{\Omega}) \cap \stackrel{\circ}{W_{\mathrm{loc}}^{1, q}}(\bar{\Omega}) \cap L^{q_{0}}(\Omega), \\
L u \in L^{p}(\Omega),
\end{array}\right.
$$

where $q \in] 1, p]$ and $q_{0} \in[1, p]$, then $u$ belongs to $W^{2, p}(\Omega)$.

Proof. The proof follows the lines of that one of Lemma 4.2 in [4], but taking care to apply our Lemma 4.1 and Theorem 3.2 in place of Lemma 4.1 and Theorem 3.1 in [4], respectively.

\section{Uniqueness and existence results}

Now we turn to the Dirichlet problem (1.1) and we first prove the following uniqueness result.

Theorem 5.1. Assume $\left(h_{1}\right),\left(h_{2}^{\prime}\right),\left(h_{3}^{\prime}\right)$ and that

$$
\exists a_{0} \in \mathbb{R}_{+} \text {such that } a \geq a_{0} \text { a.e. in } \Omega \text {. }
$$

Moreover, if $p \leq 2$, suppose also $\left(h_{2}\right)$ (with $r>2$ ) and $\left(h_{4}\right)$. Then the problem 


$$
\left\{\begin{array}{l}
u \in W_{\mathrm{loc}}^{2, p}(\bar{\Omega}) \cap \stackrel{\circ}{W}^{1, p}(\Omega), \\
L u=0
\end{array}\right.
$$

admits only the zero solution in $\Omega$.

Proof. Note that Sobolev embedding yields $u \in C^{0}(\bar{\Omega}) \cap \stackrel{\circ}{W^{1, p}}(\Omega)$, hence $u_{\mid \partial \Omega}=0$. If $p>2$, then $\lim _{|x| \rightarrow+\infty} u(x)=0$, and so the result follows applying Corollary 4.2 in [8]. If $p \in] 1,2]$, Lemma 4.2 above guarantees that $u \in W^{2, p}(\Omega)$; so by Sobolev embedding $u$ lies in $W^{1, \tilde{p}}(\Omega)$, with $\frac{1}{\tilde{p}} \in\left[\frac{1}{p}-\frac{1}{2}, \frac{1}{2}\left[\right.\right.$. Therefore $\lim _{|x| \rightarrow+\infty} u(x)=0$, and so, also in this case, the statement follows by Corollary 4.2 in [8].

Finally, adding the following assumption on the coefficients of $L$

$\left(h_{5}\right)\left\{\begin{array}{l}\left(\alpha_{i j}\right)_{x_{h}}, a_{i} \in M_{0}^{r}(\Omega), i, j, h=1,2, \\ \text { where } r>2 \text { if } p \leq 2, r=p \text { if } p>2, \\ a=a^{\prime}+b, \text { where } a^{\prime} \in M_{0}^{p}(\Omega), b \in L^{\infty}(\Omega), b_{0}=\underset{\Omega}{\operatorname{ess} \inf b>0,} \\ g \in \operatorname{Lip}(\bar{\Omega}), g_{0}=\underset{\Omega}{\operatorname{ess} \inf } g>0,\end{array}\right.$

we are now in position to state the following uniqueness and existence result.

Theorem 5.2. Under $\left(h_{1}\right),\left(h_{3}^{\prime}\right),\left(h_{4}\right),\left(h_{5}\right)$ and (5.1), the Dirichlet problem

$$
\left\{\begin{array}{l}
u \in W^{2, p}(\Omega) \cap \stackrel{\circ}{W}^{1, p}(\Omega), \\
L u=f, \quad f \in L^{p}(\Omega)
\end{array}\right.
$$

is uniquely solvable.

Proof. One can proceed as in the proof of Theorem 4.3 in [5], but replacing the applications of Theorem 5.2 in [4] and Theorem 5.1 in [6] with the ones of previous Theorem 5.1 and Lemma 3.1, respectively.

Remark 5.3. An example of functions $a_{i j}$ verifying the assumptions $\left(h_{1}\right)$, $\left(h_{3}^{\prime}\right),\left(h_{4}\right)$ and $\left(h_{5}\right)$ of Theorem 5.2, but such that $\left(a_{i i}\right)_{x_{h}}$ do not belong to $M_{0}^{p}(\Omega)$ for any $p \in[1,+\infty[$ and $i, h=1,2$, can be obtained considering $\Omega=]-\infty, \infty[\times]-1,1\left[, \alpha_{i j}=2 \delta_{i j}\right.$ and

$$
a_{i j}=\alpha_{i j}+\frac{\sin \left(1+e^{|x|^{2}}\right)}{1+|x|} \delta_{i j}, \quad i, j=1,2 .
$$




\section{References}

[1] R. A. Adams, Sobolev Spaces, Academic Press, New York, 1975.

[2] K. Astala, T. Iwaniec and G. Martin, Pucci's conjecture and the Alexandrov inequality for elliptic PDEs in the plane, J. Reine Angew. Math., 591 (2006), 49-74.

[3] L. Caso, P. Cavaliere and M. Transirico, Solvability of the Dirichlet problem in $W^{2, p}$ for elliptic equations with discontinuous coefficients in unbounded domains, Matematiche (Catania), 57 (2002), 287-302.

[4] L. Caso, P. Cavaliere and M. Transirico, Uniqueness results for elliptic equations with VMO-coefficients, Int. J. Pure Appl. Math., 13 (2004), 499-512.

[5] L. Caso, P. Cavaliere and M. Transirico, An existence result for elliptic equations with VMO-coefficients, J. Math. Anal. Appl., 325 (2007), 1095-1102.

[6] P. Cavaliere, M. Longobardi and A. Vitolo, Imbedding estimates and elliptic equations with discontinuous coefficients in unbounded domains, Matematiche (Catania), 51 (1996), 87-104.

[7] P. Cavaliere and M. Transirico, The Dirichlet problem for elliptic equations in the plane, Comment. Math. Univ. Carolin., 46 (2005), 751-758.

[8] P. Cavaliere and M. Transirico, A strong maximum principle for linear elliptic operators, Int. J. Pure Appl. Math., to appear.

[9] F. Chiarenza, M. Frasca and P. Longo, Interior $W^{2, p}$ estimates for non divergence elliptic equations with discontinuous coefficients, Ricerche Mat., 40 (1991), 149-168.

[10] F. Chiarenza, M. Frasca and P. Longo, $W^{2, p}$-solvability of the Dirichlet problem for nondivergence elliptic equations with VMO coefficients, Trans. Amer. Math. Soc., 336 (1993), 841-853.

[11] A.V. Glushak, M. Transirico and M. Troisi, Teoremi di immersione ed equazioni ellittiche in aperti non limitati, Rend. Mat., 9 (4) (1989), 113-130.

[12] A. Maugeri, D.K. Palagachev and L.G. Softova, Elliptic and Parabolic Equations with Discontinuous Coefficients, Wyley-Vch, Berlin, 2000.

[13] C. Miranda, Sulle equazioni ellittiche del secondo ordine a coefficienti discontinui, Ann. Mat. Pura Appl., 63 (4) (1963), 353-386.

[14] C. Pucci, Equazioni ellittiche con soluzioni in $W^{2, p}, p<2$, Atti del Convegno sulle Equazioni alle Derivate Parziali (Bologna) (1967), 145148. 
[15] G. Talenti, Equazioni lineari ellittiche in due variabili, Matematiche (Catania), 21 (1966), 339-376.

[16] M. Transirico and M. Troisi, Equazioni ellittiche del secondo ordine di tipo non variazionale in aperti non limitati, Ann. Mat. Pura Appl., 152 (7) (1988), 209-226.

[17] M. Transirico, M. Troisi and A. Vitolo, Spaces of Morrey type and elliptic equations in divergence form on unbounded domains, Boll. Un. Mat. Ital., 9B (7) (1995), 153-174.

[18] M. Transirico, M. Troisi and A. Vitolo, BMO spaces on domains of $\mathbb{R}^{n}$, Ricerche Mat. 45 (1996), 355-378.

[19] C. Vitanza, $W^{2, p}$-regularity for a class of elliptic second order equations with discontinuous coefficients, Matematiche (Catania), 47 (1992), 177-186.

[20] C. Vitanza, A new contribution to the $W^{2, p}$-regularity for a class of elliptic second order equations with discontinuous coefficients, Matematiche (Catania), 48 (1993), 287-296.

Dipartimento di Matematica e Informatica

Facoltà di Scienze MM.FF.NN.

Università di Salerno, via Ponte Don Melillo

I 84084 Fisciano (SA)

Italy

(E-mail : pcavaliere@unisa.it)

(E-mail : mtransirico@unisa.it)

(Received : February 2007) 


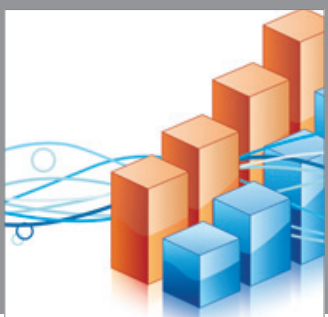

Advances in

Operations Research

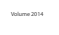

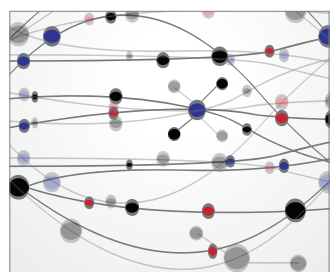

\section{The Scientific} World Journal
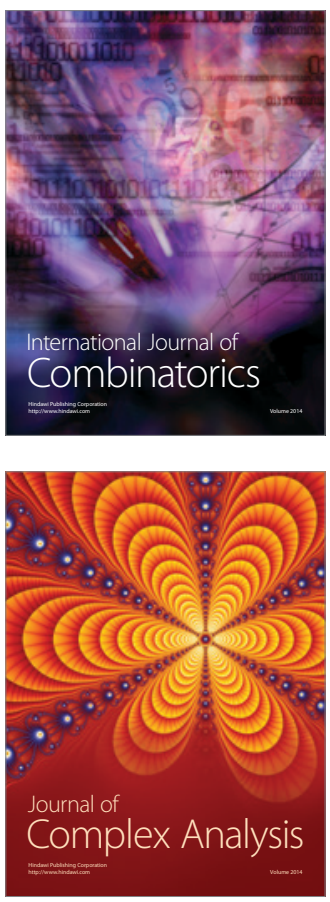

International Journal of

Mathematics and

Mathematical

Sciences
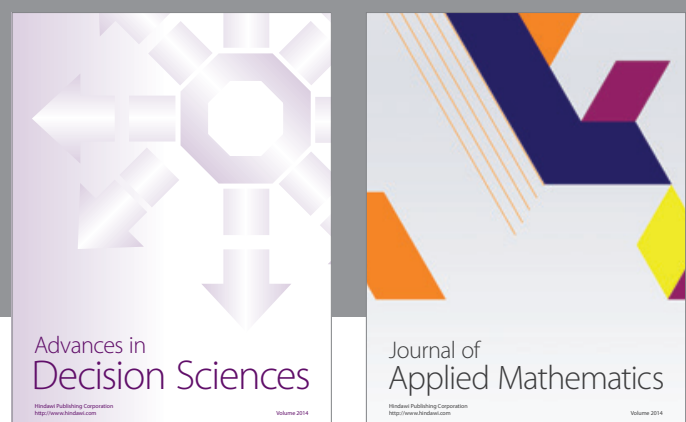

Journal of

Applied Mathematics
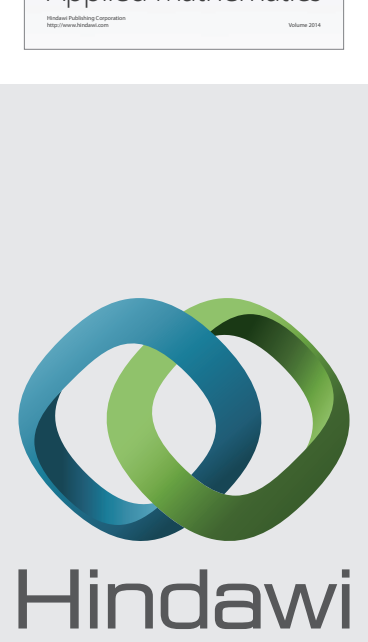

Submit your manuscripts at http://www.hindawi.com
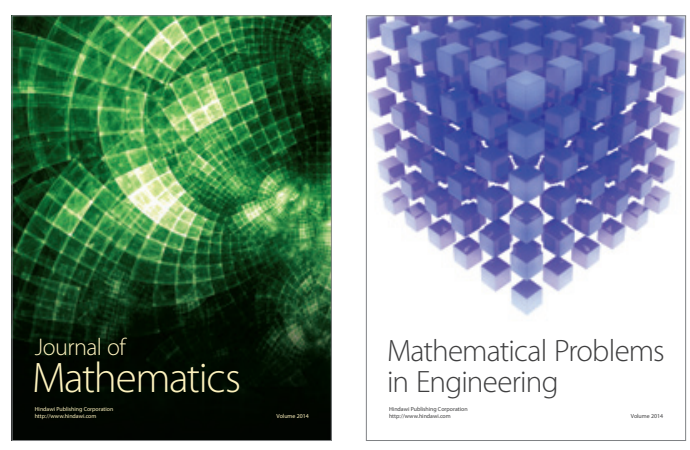

Mathematical Problems in Engineering
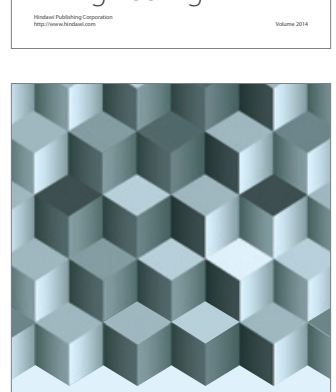

Journal of

Function Spaces
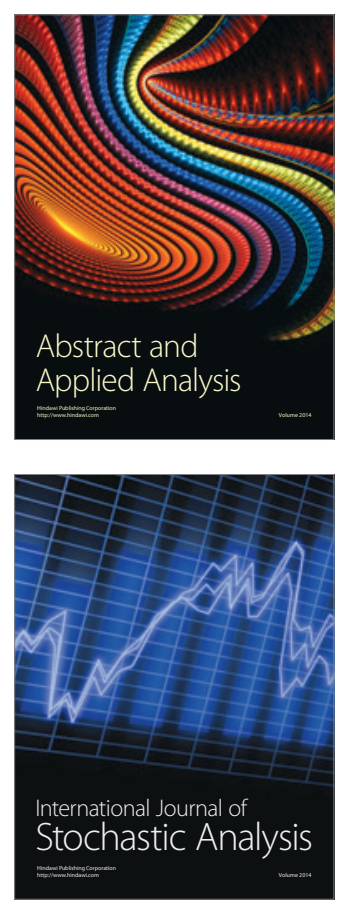

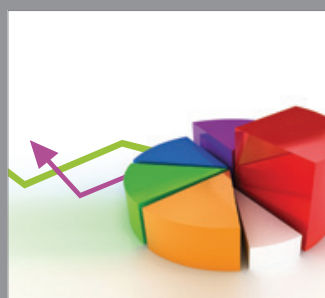

ournal of

Probability and Statistics

Promensencen
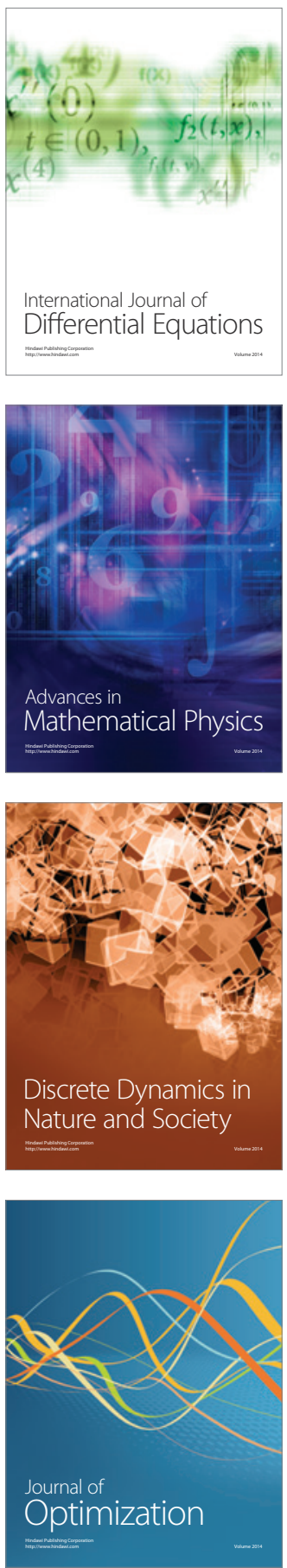